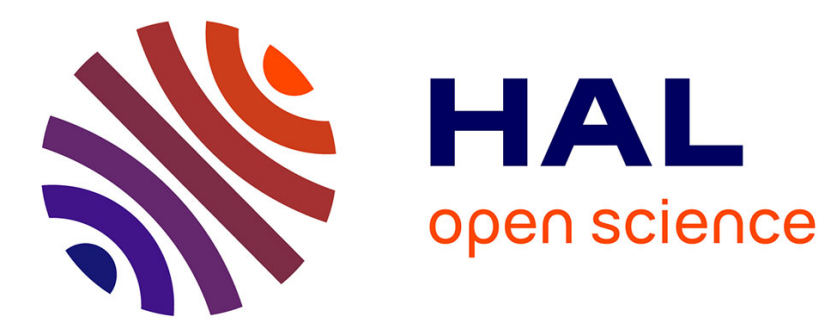

\title{
Effets Hawthorne négatifs : quels motifs aux surexpressions de la douleur?
}

Jean-Marie Berthelot, Julien Nizard, Yves Maugars

\section{To cite this version:}

Jean-Marie Berthelot, Julien Nizard, Yves Maugars. Effets Hawthorne négatifs: quels motifs aux surexpressions de la douleur?. Revue du Rhumatisme, 2019, 86, pp.134 - 138. 10.1016/j.rhum.2018.08.005 . hal-03486232

\section{HAL Id: hal-03486232 \\ https://hal.science/hal-03486232}

Submitted on 20 Dec 2021

HAL is a multi-disciplinary open access archive for the deposit and dissemination of scientific research documents, whether they are published or not. The documents may come from teaching and research institutions in France or abroad, or from public or private research centers.
L'archive ouverte pluridisciplinaire HAL, est destinée au dépôt et à la diffusion de documents scientifiques de niveau recherche, publiés ou non, émanant des établissements d'enseignement et de recherche français ou étrangers, des laboratoires publics ou privés.

\section{다)(1) $(5$}

Distributed under a Creative Commons Attribution - NonCommercial| 4.0 International 
Version of Record: https://www.sciencedirect.com/science/article/pii/S1169833018301546

Manuscript_efe4c4e28e4c809459cc9049a3a3f5b6

Effets Hawthorne négatifs : quels motifs aux surexpressions de la douleur?

Jean-Marie Berthelot (1), Julien Nizard (2), Yves Maugars (1).

1-Service de Rhumatologie, Hôtel-Dieu, CHU Nantes, 44093, Nantes Cedex 01

2-Service Douleur et Soins de Support, Hôpital Georges et René Laennec, CHU Nantes, 44093, Nantes Cedex 01

Auteur correspondant: Service de Rhumatologie, Hôtel-Dieu, CHU Nantes, Place Alexis Ricordeau, 44093 Nantes Cedex 01, France

Tel : (33)(0)2.40.08.48.22/01/25

Fax : (33)(0)2.40.08.48.30

Courriel : jeanmarie.berthelot@chu-nantes.fr 


\section{Résumé :}

En médecine, l'effet Hawthorne positif correspond à la moindre expression d'un symptôme en présence d'un évaluateur. II s'additionne au moindre ressenti que procure l'effet placebo. Quand le symptôme est au contraire surexprimé, consciemment ou inconsciemment, il s'agit d'un effet Hawthorne négatif, à distinguer des effets nocebo, durant lesquels le symptôme est réellement plus ressenti. Un exemple d'effet Hawthorne négatif est celui des patients évaluant calmement leurs douleurs à onze sur dix. Les motifs d'effets Hawthorne négatifs sont nombreux: souhait d'être davantage considéré(e), ou pris(e) en charge prioritairement ; craintes de ne pas valider les critères d'obtention d'un nouveau traitement ou d'intégration dans un essai thérapeutique; conformisme culturel ou de circonstance, comme de ne pas annuler une intervention chirurgicale lorsqu'une amélioration survient in extremis; disease mongering, ou stratégie de Knock; désir d'être pris au sérieux par son entourage; recherche de bénéfices secondaires; utilisation de l'évaluation pour exprimer sa frustration d'être malade, ou son dépit de ne plus être choyé; décalage entre les attentes d'un soulagement complet et l'efficience réelle des traitements ; majoration des douleurs, à l'origine de la démonétisation de leur chiffrage, par anxiété, culpabilité, dépression, nervosisme, catastrophisme, kinésiophobie, ou du fait d'évaluations répétées avec des procédés comportant des suggestions négatives, comme l'évocation de la pire douleur imaginable. La succession de forts effets Hawthorne négatifs avant prescription, puis de forts effets Hawthorne positifs après introduction des thérapeutiques, pourrait, plus que l'effet placebo, expliquer une bonne part des améliorations observées dans le traitement des douleurs.

Mots-clés: douleur; Hawthorne ; négatif ; placebo ; nocebo ; surestimation ; surévaluation ; majoration; échelle de douleur ; open-hidden. 
L'effet Hawthorne correspond aux situations dans lesquelles les résultats d'une expérience sont modifiés par le fait que les sujets ont conscience d'être évalués. Ceci se traduit généralement par une plus grande motivation, et/ou un souhait de se conformer aux attentes des évaluateurs [1-2].

En médecine, l'effet Hawthorne est souvent amalgamé à l'effet placebo [3], mais les mécanismes en sont différents. L'effet placebo désigne l'amélioration réellement ressentie lors de la prise d'un simulacre de traitement, grâce à la libération de neuromédiateurs qui vont inhiber le symptôme (dont des endorphines), ou le rendre moins désagréable (dont la dopamine)[4].

L'effet Hawthorne (positif) correspond à une moindre expression du symptôme selon les circonstances, ou l'évaluateur(trice): par exemple, un patient hospitalisé peut, en présence d'une étudiante en médecine charmante, coter sa douleur à 4 sur 10 (minimisation), alors que quelques minutes plus tôt il avait évalué cette même douleur à 6 sur 10 à la demande d'un interne taciturne (exagération). Cet effet Hawthorne peut être particulièrement marqué, et plus important que l'effet placebo stricto sensu, quand la variable étudiée est subjective.

L'effet Hawthorne peut expliquer pourquoi des « effets placebos » (qui sont en fait surtout des effets Hawthorne) peuvent durer des mois, comme après pratique d'un simulacre de vertébroplastie (sans injection de ciment). Des études en double-insu ont montré que chez les patientes n'ayant eu qu'une fausse injection de ciment, la douleur décrite était passée presque immédiatement de 6 à 3 , et que cette cotation était restée la même pendant les 3 mois suivants, alors que des patientes contrôles (n'ayant même pas eu de simulacre de vertébroplastie) cotaient encore leurs douleurs à 5 à 3 mois [5], alors qu'une libération d'endorphine ou de dopamine ne peut perdurer trois mois.

La plupart des travaux menés sur l'effet Hawthorne n'ont considéré que les améliorations induites, et la possibilité d'effets Hawthorne négatifs n’a pas été encore évoquée.

Pourtant, la conscience par les patients d'être observés/évalués peut aussi induire une majoration de l'expression de divers symptômes. Si ces aggravations sont souvent attribuées à des effets nocebo [67], cette dénomination n'est pas toujours appropriée. En effet, les effets nocebo lato-sensu correspondent à l'aggravation réelle de la douleur, du fait de la libération de différents 
neuromédiateurs comme la cholécystokinine (laquelle antagonise aussi les effets placebos) [8-9], de la même manière que les effets nocebo stricto sensu correspondent à l'induction d'effets indésirables réellement perçus dans les suites d'une suggestion négative.

L'importance des effets Hawthorne négatifs est quantifiable lors des administrations de traitements en intraveineux selon la méthodologie de type open-hidden [10]. Tous les patients y reçoivent un vrai traitement présenté comme efficace (par exemple de la morphine) au moyen d'une seringue électrique télécommandée à distance, sans qu'ils puissent savoir quand l'administration du produit débute ou est suspendue. Une moitié est laissée dans l'ignorance du moment où le produit est administré, tandis qu'on dit à l'autre quand précisément l'antalgique est administré, et quand précisément on l'arrête. La comparaison des courbes obtenues permet de remarquer, dans le groupe des patients informés, d'une part une amélioration immédiate de la douleur, dès l'annonce que le produit a été initié (versus une amélioration plus lente dans le groupe en insu), soit un effet Hawthorne positif quasiment instantané. On peut observer aussi une remontée très rapide de la douleur quand la fin de l'administration est dévoilée, phénomène non observé chez les patients restant en insu du moment d'arrêt du traitement [10]. Ce rebond a été interprété comme la résultante d'un effet 'anti-placebo' dû à l'attente du patient d'une perte d'effet immédiate du traitement (fin de la perfusion). Même s'il faudrait idéalement disposer d'une IRM fonctionnelle pour I'affirmer [11], la remontée quasi-instantanée des chiffres de la douleur juste après l'arrêt du traitement s'expliquerait bien mieux par un effet Hawthorne négatif dans le groupe informé, que d'un effet « anti-placebo ».

La ré-ascension immédiate des chiffres de douleur dans le groupe informé n'est de fait pas cohérente avec la durée de l'effet pharmacologique de la morphine, lequel se prolonge pendant une à trois heures. De plus, un effet anti-placebo aussi important cadrerait mal avec le fait que le profil psychologique des patients bons répondeurs à l'effet placebo diffère souvent nettement de celui des patients développant des effets nocebo. La majoration moyenne des douleurs de 0,74 points sur une 
échelle de 0 à 10 après simple suggestion négative [12] (soit très proche du seuil de changement cliniquement significatif de 1,0 [13]) (Encadré 1) conforte cette hypothèse.

La réalité des effets Hawthorne négatifs est bien illustrée par l'exemple des patients estimant avec un visage détendu que leur douleur est à « onze sur dix ».

Le but de cet article est de tenter de lister les principaux motifs et circonstances pouvant inciter des patients à majorer, consciemment ou inconsciemment, leurs symptômes. Ce sujet n'est pas futile, car dans de nombreux contextes, dont le traitement de la douleur, la somme des effets Hawthorne (secondairement positifs, mais préalablement négatifs) peut être aussi plus importante que l'efficacité réelle de certaines thérapeutiques [14].

\section{1-Effets Hawthorne négatifs par conformisme}

Dans les effets Hawthorne positifs le sujet observé tend à satisfaire les attentes d'un bon résultat (dans l'expérience princeps, la productivité des ouvrières d'une usine éponyme s'était accrue durant une inspection destinée initialement à savoir si un meilleur éclairage allait majorer leur efficience, les évaluateurs ayant au final remarqué que la productivité était restée meilleure à la fin de la période d'observation, même quand, à l'insu des employées, le niveau d'éclairage avait été ramené à celui utilisé avant le test)[1].

Il peut arriver toutefois que les évaluateurs espèrent un résultat plutôt négatif, comme la présence d'un nouveau signe pouvant aider le praticien à poser un diagnostic, quand celui-ci reste encore incertain. Certains patients, et en particulier les hypochondriaques, peuvent alors amplifier un symptôme suggéré par le médecin, ce qui peut aboutir à un diagnostic construit. Ceci peut survenir d'autant plus facilement quand la personne interrogée a déjà fait des recherches sur internet [15], et sait qu'il s'agit d'un des symptômes possibles de la pathologie qu'il craint (voire espère) avoir. Un interrogatoire sourcilleux de ce type de personnalité, comme dans la célèbre pièce de Jules Romain, 'Knock', peut renforcer la conviction de tels patients qu'ils souffrent vraiment de cette entité 
pathologique. S'en rapproche le disease mongering, qui correspond à la genèse de nouvelles maladies sur la combinaison de signes triviaux, ou à l'abaissement des seuils de symptômes pour poser un diagnostic ou décider d'un traitement ('stratégies de Knock').

Un autre exemple de conformisme source d'effets Hawthorne négatifs est celui des personnes pour lesquelles un rendez-vous de bloc opératoire a été retenu pour une pathologie douloureuse. Certains patients redevenus presque indolents peu de temps avant l'intervention peuvent alors ne pas oser en informer le chirurgien (du fait de la difficulté que celui-ci a eue pour fixer une date), et surévaluer leur douleur résiduelle si on les interroge alors, afin de ne pas placer l'équipe chirurgicale dans la situation de devoir annuler in extremis l'opération. L'évaluation de la douleur rendue aux chirurgiens peut dans ce contexte être supérieure à celle rendue aux infirmières [16].

L'appartenance à certaines cultures paraît aussi influencer la manière de coter et d'exprimer la douleur [17], avec la réserve d'une génétique aussi différente [18].

\section{2-Effets Hawthorne négatifs par souci d'être pris(e) en charge plus vite}

Une majoration plus ou moins consciente dans l'expression de la douleur peut résulter de la crainte de ne pas être considéré(e) comme prioritaire lorsqu'existe une compétition dans l'accès au soin. Ceci peut survenir dans des services d'urgence ou des structures de long séjour, où la carence relative en personnel peut inciter certains patients à une exagération dans l'expression de leur douleur. Cela peut aussi survenir dans des centres de traitement de la douleur, au sein desquels les délais de prise en charge peuvent être importants.

\section{3-Effets Hawthorne négatifs liés à la nécessité de satisfaire des critères d'activité}

En pratique quotidienne, l'obtention de certains traitements novateurs est théoriquement conditionnée à des niveaux minimums d'activité de la pathologie (exemple du score BASDAI devant 
être supérieur à 4/10 dans le contexte des spondyloarthrites pour justifier de l'introduction d'un antiTNF alpha) [19]. Ceci peut induire une majoration des doléances, si cette notion de seuil est sur des patients.

La publicité faite à un nouveau procédé thérapeutique peut aussi inciter certaines personnes à en espérer un encore meilleur résultat qu'avec le traitement en cours. Peut s'en suivre une péjoration artificielle de leur état présent pour inciter le médecin à leur délivrer le traitement souhaité, qui peut être une chirurgie [20].

Dans le contexte des essais cliniques, il peut arriver qu'après de chronophages visites de préinclusion, et le recueil parfois difficile du consentement de participation, l'inclusion soit au dernier moment compromise, si, du fait d'une amélioration intercurrente, les patients n'évaluent plus leur pathologie comme assez active sur une échelle verbale ou analogique. II est possible que certains patients, informés ou non par les médecins de cette nécessité, surestiment alors temporairement leurs plaintes pour pouvoir être randomisés et peut-être bénéficier du traitement à l'essai. Ceci pourrait contribuer à expliquer des améliorations très rapides, enregistrées dès la première évaluation, au moment de la première administration du produit. II a aussi été souligné que le seul recrutement dans l'essai pouvait suffire à majorer l'évaluation initiale [2,21].

\section{4-Effets Hawthorne négatifs par crainte de ne pas être pris(e) au sérieux}

Une majoration dans l'expression des douleurs peut découler de la crainte que celles-ci soient minimisées par l'entourage, médical, ou non médical (lieux de travail, famille). Un exemple trivial en est donné par le comportement parfois spectaculaire de certains joueurs de football pour attirer l'attention de l'arbitre, que l'on n'observe guère sur un terrain de rugby, où les affrontements sont souvent plus rugueux, mais ne sont pas sanctionnés de la même manière.

\section{5-Effets Hawthorne négatifs liés à la recherche d'un bénéfice secondaire}


Une exagération des plaintes peut aussi témoigner d'une recherche de réparation en cas d'accident de travail, ou du maintien d'une situation jugée comme avantageuse par le patient (recherche de compensations financières, poursuite d'un arrêt de travail, d'une aide sociale, ou quête d'une situation d'invalidité), surtout quand celle-ci est conditionnée par un niveau minimum d'activité de la pathologie. Cette variété d'effet Hawthorne négatif a été dénommée malingering (situation intermédiaire entre la simulation et la sinistrose) [22].

\section{6-Effets Hawthorne négatifs visant à sanctionner l'évaluateur}

La frustration de certains patients de ne pas avoir obtenu le niveau de soulagement escompté peut aboutir à la majoration de leurs doléances pour souligner l'inefficience de la prise en charge, même quand les soignants ont prodigué les meilleurs soins possibles. Cette attitude peut être encouragée par les questionnaires de satisfaction remis aux patients lors de leur sortie des services (qui peuvent inciter certains d'entre eux à se comporter comme des consommateurs revendicateurs, ou des patients " passifs-agressifs »), ou par des ouvrages polémiques taxant les professionnels de soins de «brutes en blancs ».

Paradoxalement, même quand le contact avec les soignants a été très bon, comme lors d'un essai clinique, des patients peuvent inconsciemment manifester après leur sortie de l'étude leur dépit de ne plus être aussi bien entourés-accompagnés, en scorant davantage leurs douleurs que durant l'essai [23]. Un suivi juste après la fin d'un essai a par exemple montré une dégradation rapide du score du HAQ (de près de la moitié), et une remontée de $23 \%$ à $44 \%$ des douleurs et jugements globaux des patients [23]. Il pourrait toutefois s'agir là plutôt d'un anti-effet Hawthorne positif que d'un effet Hawthorne négatif.

\section{7-Effets Hawthorne négatifs par interférences de l'évaluation avec diverses émotions}


Le niveau d'expression d'une douleur peut être majoré par diverses émotions.

Il s'agit d'abord d'une anxiété, d'une part de souffrir davantage, d'autre part de ne plus être en mesure de faire face à son quotidien du fait des limitations induites par la douleur. Dans ce cas, la coprescription d'un anxiolytique pourrait être aussi, voire plus, efficace que celle d'antalgiques, et prévenir leur mésusage [24]. Une attitude rassurante de la part des soignants devrait rester le meilleur moyen d'éviter une majoration dans l'expression de la douleur induite par l'anxiété. Une analyse précoce des convictions erronées des patients quant à la sévérité de leurs troubles peut ainsi minimiser l'expression de la douleur du fait, non seulement de la disparition de l'effet Hawthorne négatif, mais aussi de l'induction d'un vrai effet placebo, en transformant les auto-suggestions négatives des patients en conviction d'une amélioration à venir [25].

Ce remède-médecin (ou paramédical) reste toutefois moins efficace quand une dépression ou un névrosisme [26] contribue à la péjoration de la douleur, ou quand le sujet craint de perdre son emploi du fait d'une baisse de ses performances. Pour interpréter un score de douleur en routine et guider les premières thérapeutiques, en particulier lors de l'admission des patients dans un service, il serait donc bienvenu, si les conditions s'y prêtent, de réaliser simultanément une évaluation de leur état psychologique, par un score HAD [27], plus facilement qu'avec un score de névrosisme, tel que celui contenu dans le questionnaire Bigfive [26], tout en s'enquérant de leur situation professionnelle.

II peut s'agir ensuite d'une culpabilité de souffrir [28], quand la douleur pénalise indirectement l'entourage (surcharge de travail ou épuisement des proches/aidants, annulation de vacances familiales...). Cette culpabilité est d'autant plus importante quand les patients ont la conviction de n’avoir pas fait « ce qu'il fallait » pour diminuer cette douleur. Cette faille a été exploitée par certains slogans publicitaires d'antalgiques (« défense d’avoir mal »), qui visaient aussi à pousser les médecins à plus prescrire. 
Un autre cofacteur majeur d'effet Hawthorne négatif pourrait être la frustration collective générée par les sur-promesses faites par certains responsables politiques, et/ou les médias, d'un soulagement complet des douleurs, en décalage marqué avec les données d'evidence-based medicine. Les publicités des firmes sont souvent plus prudentes, mais certaines véhiculent un message subliminal aussi frustrant, voire culpabilisant, comme l'image (pour de présumés anti-arthrosiques) d'une femme de 70 ans réalisant sur son plancher un grand écart avec un sourire radieux.

II s'agit aussi du catastrophisme, résultante de trois dimensions (rumination, sentiment d'impuissance, et amplification des symptômes) [29]. Bien que ce dernier composant soit presque synonyme d'effet Hawthorne négatif, les deux autres dimensions du catastrophisme peuvent y prédisposer.

Même si ceci reste à démontrer, il est enfin possible que la kinésiophobie [30] induise chez certains patients des effets Hawthorne négatifs, "prétextes" à se soustraire aux efforts. Certaines améliorations constatées après physiothérapie pourraient donc aussi relever, au moins partiellement, de la correction de tels effets Hawthorne négatifs.

\section{8-Effets Hawthorne négatifs induits par la répétition des évaluations}

Malgré l'usage (et le mésusage) des antalgiques induits par les campagnes de sensibilisation au traitement de la douleur aux USA, puis dans les autres pays, le niveau moyen de douleurs dans la population ne semble pas avoir baissé, trois quarts des patients sous opioïdes forts se disant toujours très douloureux [31]. Ceci illustre à nouveau l'efficacité plus faible des antalgiques que celle présumée par le grand public, et une bonne partie du corps médical-paramédical (croyance relayée parfois par des autorités de santé n'ayant pas bien évalué la réelle efficience et dangerosité des molécules promues, sur des données scientifiques).

Ce constat pourrait toutefois traduire aussi une tendance des patients à coter plus sévèrement leurs douleurs, d'origine multifactorielle. 
Une première raison pourrait être l'habitude prise d'un grand confort matériel : le caractère douillet des modes de vie moderne peut aussi induire de la part de certains individus un comportement similaire.

Une seconde raison pourrait être l'algophobie induite par les vigoureuses campagnes de marketing menées depuis trente ans, tant auprès des médecins que des autorités sanitaires et du grand public, pour substituer à l'opiophobie du passé une opiophilie légitimée par une dramatisation de la douleur.

Un dernier cofacteur à cette inflation pourrait être la répétition des évaluations de la douleur, d'abord institutionnalisée aux USA, puis dans d'autres pays : la présentation pluriquotidienne aux patients hospitalisés d'une échelle analogique comportant un extrême défini comme "la pire douleur imaginable », pourrait avoir des effets néfastes de suggestion négative. De la même manière que l'injonction « ne pensez pas à un éléphant » réveille immédiatement dans les circuits neuronaux l'image d'un éléphant, l'évocation de " la pire douleur imaginable ", pourrait contribuer à entretenir une inquiétude latente à l'égard d'une telle évolution chez beaucoup de patients déjà douloureux. Elle pourrait aussi contribuer à inhiber la survenue d'un effet placebo (que l'anxiété atténue, voire abolit), et à induire des effets Hawthorne négatifs. Ceci est d'autant plus probable qu'une des règles de base des thérapeutiques dites psychocorporelles (comme la sophrologie, l'autohypnose ou la méditation) est au contraire d'évacuer la douleur de son champ de conscience [32], ou d'en limiter l'impact en termes cognitifs et émotionnels. Certaines injonctions illustrent le risque de devenir plus vulnérable lorsqu'on s'apitoie par excès sur soi-même (un royal contre-exemple étant le «never explain, never complain » de la reine Victoria à son fils, le futur Edouard VII). Beaucoup de rhumatisants adoptent d'ailleurs comme première règle de coping d'éviter de trop se plaindre, à l'inverse du penchant à la rumination des catastrophistes [33].

Mériteraient donc d'être menées des études randomisées en insu comparant l'évolution dans le temps des niveaux moyens de douleurs de patients souffrant d'un même type et sévérité de 
pathologies, selon qu'on les oblige à s'évaluer de manière pluriquotidienne, ou seulement au début et à la fin de la prise en charge. II est probable que la première attitude aboutit à une plus grande consommation d'antalgiques, mais reste à démontrer qu'au final la qualité du soulagement est meilleure qu'avec des évaluations moins incisives et répétées.

S'il ne faut pas tomber dans l'attitude extrême d'accuser le thermomètre (le dolorimètre) d'être responsable de la fièvre (la douleur), il convient donc de s'interroger sur les conséquences d'une répétition excessive des mesures [34]. Celle-ci pourrait en effet contribuer à une sensibilisation psychologique à la douleur se rajoutant à la sensibilisation neurologique centrale déjà à l'œuvre chez certains patients [35]. Ces excès de mesure pourraient au final avoir plus profité aux firmes qu'aux patients et médecins, même en faisant abstraction des centaines de milliers de morts par overdoses d'opiacés, qu'un mésusage des « pain-killers » a induit aux USA [36-37].

L'induction d'effets Hawthorne négatifs du fait d'évaluations trop répétées de la douleur pourrait aussi être une limite aux autoévaluations à domicile des rhumatismes inflammatoires par les patients eux-mêmes, par exemple au moyen du score RAPID3 (somme de la douleur sur une échelle analogique de 0 à 10 , de l'activité de la maladie sur une échelle de 0 à 10 , et du score fonctionnel $H A Q)$, malgré les avantages de ce score, dont sa rapidité, sa simplicité, et la possibilité de l'utiliser dans toutes les pathologies de l'appareil locomoteur [38].

\section{Conclusion}

La possibilité d'induction d'effets Hawthorne négatifs rappelle que: 1-évaluer un patient n'est pas une activité neutre, des mesures répétées pouvant influencer les résultats ; 2-la suggestion joue un rôle non seulement dans le ressenti des symptômes (effet placebo), mais aussi dans leur expression (effets Hawthorne positifs, à utiliser [39], et négatifs, à combattre) ; 3-des effets Hawthorne négatifs pourraient altérer le coping [33]. 
Certaines améliorations observées dans les essais cliniques tiennent à la somme d'effets Hawthorne initialement négatifs (majoration à l'inclusion), puis positifs (minoration une fois sous traitement), ceci expliquant un différentiel d’amélioration souvent plus important qu'attendu [40] : dans un travail récent mené dans des services d'urgences New-Yorkais ayant étudié en double-insu la combinaison de paracétamol et d'ibuprofène à la combinaison de paracétamol et d'oxycodone, les scores de douleurs rendus ont baissé à deux heures de manière équivalente dans les deux bras, passant de 8,9 à 4,3 dans le bras ibuprofène et de 8,7 à 4,4 dans le bras oxycodone [41], soit une amélioration de la douleur de 4,5 sur 10 . Pourtant, les méta-analyses des études randomisées menées versus placebo avec les morphiniques ont conclu que le différentiel réel de ces traitements par rapport à un placebo était de seulement 1,2 sur 10 pour les classes III (soit 0,36 de mieux que le paracétamol per os (Encadré 1) [42]), aucune différence n'ayant même été notée dans d'autres études randomisées comparant aux urgences morphine IV et paracétamol IV [43]. De ce fait, près des trois-quarts de l'amélioration de la douleur $(3,3 / 4,5)$ observée dans cet essai résultait sans doute de la somme d'effets Hawthorne négatifs marqués à l'arrivée aux urgences (anxiété et crainte de ne pas être pris en charge en priorité, induisant une sur-cotation de la douleur à près de 9 sur 10), puis d'effets placebo et Hawthorne positifs.

Si les industriels du médicament peuvent profiter de cette séquence d'un effet Hawthorne négatif avant traitement, à un effet Hawthorne positif une fois le traitement administré, qui renforce la conviction générale d'une forte efficacité de leurs molécules, ni les médecins, ni leurs patients, n'ont intérêt à ce que les douleurs avant traitements soient surévaluées. Ceci contribuerait à une inflation dans l'expression des douleurs et à une majoration du catastrophisme [44] (lequel peut gommer une grande partie des améliorations induites par les vrais progrès thérapeutiques [29]), voire à un mésusage des antalgiques [43].

De même, si une mesure de l'activité d'un rhumatisme, et des douleurs qu'il induit, est indispensable dans les essais cliniques, y compris par des questionnaires rétrospectifs destinés à capter rétroactivement les poussées douloureuses [45], il n'est sans doute pas anodin d'inciter les patients à 
quantifier trop souvent leurs douleurs. II est par contre sans doute bienvenu de demander aux patients hospitalisés s'ils sont " assez soulagés ", plutôt que de leur évoquer plusieurs fois par jour «la pire douleur imaginable» et de lutter contre certains effets Hawthorne négatifs par des suggestions verbales optimistes [46].

Conflits d'intérêt : aucun 


\section{Références}

1-Berthelot JM, Le Goff B, Maugars Y. The Hawthorne effect: stronger than the placebo effect? Joint Bone Spine 2011;78:335-6.

2-Sedgwick P, Greenwood N. Understanding the Hawthorne effect. BMJ 2015;351:h4672. doi: 10.1136/bmj.h4672.

3-Abhishek A, Doherty M. Understanding placebo effects in rheumatology. Joint Bone Spine 2015;82:222-4. Joint Bone Spine 2015;82:222-4.

4-Dodd S, Dean OM, Vian J, et al. A Review of the Theoretical and Biological Understanding of the Nocebo and PlaceboPhenomena. Clin Ther 2017;39:469-76.

5-Kallmes DF, Comstock BA, Heagerty PJ, Turner JA, Wilson DJ, Diamond TH, Edwards R, Gray LA, Stout L, Owen S, Hollingworth W, Ghdoke B, Annesley-Williams DJ, Ralston SH, Jarvik JG. A randomized trial of vertebroplasty for osteoporotic spinal fractures. N Engl J Med. 2009;361:569-79.

6-Rossettini G, Carlino E, Testa M. Clinical relevance of contextual factors as triggers of placebo and nocebo effects in musculoskeletal pain. BMC Musculoskelet Disord 2018;19:27. doi: 10.1186/s12891018-1943-8

7-Colloca L. Nocebo effects can make you feel pain. Science 2017; 358(6359):44. doi: 10.1126/science.aap8488.

8-Frisaldi E, Piedimonte A, Benedetti F. Placebo and nocebo effects: a complex interplay between psychological factors and neurochemical networks. Am J Clin Hypn 2015;57:267-84.

9-Aslasksen PM, Zwrg ML, Eilertsen HI, et al. Opposite effects of the same drug: reversal of topical analgesia by nocebo information. Pain 2015;156:39-46.

10-Benedetti F, Maggi G, Lopiano L et al. Open versus hidden medical treatments: the patient's knowledge about a therapy affects the therapy outcome. Prevention \& Treatment 2003; 6. Article ID 1a. <http://psycnet.apa.org/journals/pre/6/1/1a.html>

11-Atlas LY, Whittington RA, Lindquist MA, et al. Dissociable influences of opiates and expectations on pain. J Neurosci 2012;32:8053-64.

12-Busse JW ; Bartlett SJ, Dougados M, et al. Optimal Strategies for Reporting Pain in Clinical Trials and Systematic Reviews: Recommendations from an OMERACT 12 Workshop. J Rheumatol 2015;42:1962-70.

13-Madden VJ, Harvie DS, Parker R, et al. Can Pain or Hyperalgesia Be a Classically Conditioned Response in Humans? A Systematic Review and Meta-Analysis. Pain Med 2016;17:1094-111.

14-Coutaux A. Non-pharmacological treatments for pain relief: TENS and acupuncture. Joint Bone Spine 2017;84:657-661

15-Crichton F, Petrie KJ. Accentuate the positive: Counteracting psychogenic responses to media health messages in the age of the Internet. J Psychosom Res 2015;79:185-9.

16-Martin KD, Van Buren JP, Wake J, et al. Comparison of Visual Analog Pain Score Reported to Physician vs Nurse. Foot Ankle Int 2017 ; 1071100717740584 . doi: 10.1177/1071100717740584. 
17-Genêt $\mathrm{F}$, Autret $\mathrm{K}$, Roche $\mathrm{N}$, et al. Comparison of the repercussions of cLBP in four Frenchspeaking countries. Ann Phys Rehabil Med 2009;52:717-28.

18-Aufiero M, Stankewicz H, Quazi S, et al. Pain Perception in Latino vs. Caucasian and Male vs. Female Patients: Is There Really a Difference? West J Emerg Med 2017;18:737-42.

19-Dernis E, Lavie F, Pavy S, et al. Clinical and laboratory follow-up for treating and monitoring patients with ankylosing spondylitis: development of recommendations for clinical practice based on published evidence and expert opinion. Joint Bone Spine 2007;74:330-7.

20-Razmjou H, Schwartz CE, Holtby R. The impact of response shift on perceived disability two years following rotator cuff surgery. J Bone Joint Surg Am 2010;92:2178-86.

21-Benedetti F, Carlino E, Piedimonte A. Increasing uncertainty in CNS clinical trials: the role of placebo, nocebo, and Hawthorne effects. Lancet Neurol 2016;15:736-47.

22-Wygant DB, Arbisi PA, Bianchini KJ, et al. Waddell non-organic signs: new evidence suggests somatic amplification among outpatient chronic pain patients. Spine J 2017;17:505-510.

23-Wolfe F, Michaud K. The Hawthorne effect, sponsored trials, and the overestimation of treatment effectiveness. J Rheumatol. 2010;37:2216-20

24-Feingold D, Brill S, Goor-Aryeh I, et al. Misuse of prescription opioids among chronic pain patients suffering from anxiety: A cross-sectional analysis. Gen Hosp Psychiatry 2017;47:36-42.

25-Koban L, Jepma M, Geuter S, et al. What's in a word? How instructions, suggestions, and social information change pain and emotion. Neurosci Biobehav Rev 2017; 81(Pt A):29-42.

26-Bucourt E, Martaillé V, Mulleman D, et al. Comparison of the Big Five personality traits in fibromyalgia and other rheumatic diseases. Joint Bone Spine 2017;84:203-7.

27-Carmona-Terés V, Moix-Queraltó J, Pujot-Ribeira E, et al. Understanding knee osteoarthritis from the patients' perspective: a qualitative study. BMC Musculoskelet Disord $2017 ; 18: 225$. doi: 10.1186/s12891-017-1584-3.

28-Zigmond AS, Snaith RP. The hospital anxiety and depression scale. Acta Psychiatr Scand1983;67:361-70.doi:10.1111/j.1600-0447.1983.tb09716.x [PubMed]

29-Penhoat M, Saraux A, Le Goff B, et al. High pain catastrophizing scores in one-fourth of patients on biotherapy for spondylarthritis or rheumatoid arthritis. Joint Bone Spine 2014;81:235-9.

30-Perrot S, Trouvin AP, Rondeau V, et al. Kinesiophobia and physical therapy-related pain in musculoskeletal pain: A national multicenter cohort study on patients and their general physicians. Joint Bone Spine 2018;85:101-7.

31-Fredheim OM, Mahic M, Skurtveit S, et al. Chronic pain and use of opioids: a population-based pharmacoepidemiological study from the Norwegian prescription database and the Nord-Trøndelag health study. Pain. 2014;155:1213-21.

32-Ardigo S, Herrmann FR, Moret V, et al. Hypnosis can reduce pain in hospitalized older patients: a randomized controlled study. BMC Geriatr. 2016;16:14.

33-Bailly F, Foltz V, Rozenberg S, et al. Development and validation of a questionnaire categorizing behavioral strategies in patients with chronic low back pain. Joint Bone Spine 2017;84:725-31. 
34-Bailly F, Fautrel B, Gossec L. Pain assessment in rheumatology-how can we do better ? A literature review. Joint Bone Spine. 2016;83:384-8.

35-Trouvin AP, Perrot S. Pain in osteoarthritis. Implications for optimal management. Joint Bone Spine 2017 Sep 6. pii: S1297-319X(17)30156-2. doi: 10.1016/j.jbspin.2017.08.002.

36-deShazo R, Johnson M, Eriator I, et al. Backstories on the U.S. Opioid Epidemic Good Intentions Gone Bad, an Industry Gone Rogue and Watch Dogs Gone to Sleep. Am J Med 2018 Jan 31. pii: S0002-9343(18)30084-6. doi: 10.1016/j.amjmed.2017.12.045.

37-Helmerhorst GT, Teunis T, Janssen SJ, et al. An epidemic of the use, misuse and overdose of opioids and deaths due to overdose, in the United States and Canada: is Europe next? Bone Joint J 2017;99-B(7):856-64.

38-Pincus T, Sokka T. Quantitative clinical rheumatology: "keep it simple, stupid": MDHAQ function, pain, global, and RAPID3 quantitative scores to improve and document the quality of rheumatologic care. J Rheumatol 2009;36:1099-100.

39-Usichenko $\mathrm{TI}$, Julich $\mathrm{A}$, Wendt $\mathrm{M}$. 'Yes, we can' utilize the Hawthorne effect to improve postoperative analgesia. Br J Anaesth 2013;110:659.

40-Vase L, Petersen GL, Riley $\mathrm{JL} 3^{\text {rd }}$, et al. Factors contributing to large analgesic effects in placebo mechanism studies conducted between 2002 and 2007. Pain 2009;145:36-44.

41-Chang AK, Bijur PE, Esses D, et al. Effect of a Single Dose of Oral Opioid and Nonopioid Analgesics on Acute Extremity Pain in the Emergency Department: A Randomized Clinical Trial. JAMA. 2017;318:1661-1667

42-Reinecke $\mathrm{H}$, Weber $\mathrm{C}$, Lange $\mathrm{K}$, et al. Analgesic efficacy of opioids in chronic pain: recent metaanalyses. Br J Pharmacol. 2015;172:324-33.

43-Berthelot JM, Darrieutort-Lafitte C, Le Goff B, et al. Strong opioids for noncancer pain due to musculoskeletal diseases: Not more effective than acetaminophen or NSAIDs. Joint Bone Spine. 2015;82:397-401.

44-Lecorneu J, Verhoeven F, Chouk M, et al. Correlation between catastrophizing and Lequesne index in case of osteoarthritis of the knee: A prospective study. Joint Bone Spine 2017 Oct 31. pii: S1297319X(17)30188-4. doi: 10.1016/j.jbspin.2017.10.002.

45-Berthelot JM, De Bandt M, Morel J, et al. A tool to identify recent or present rheumatoid arthritis flare from both patient and physician perspectives: the 'FLARE' instrument. Ann Rheum Dis. 2012;71:1110-6.

46-Bartels DJP, van Laarhoven AIM, Stroo M, et al. Minimizing nocebo effects by conditioning with verbal suggestion: A randomized clinical trial in healthy humans. PLoS One 2017;12: e0182959. 


\section{Encadré 1}

Sur une échelle de 0 à 10, baisse réelle moyenne de la douleur induite par diverses thérapeutiques antalgiques par rapport à un placebo dans les méta-analyses, à comparer à la majoration de la douleur par effets Hawthorne-placebo négatifs seulement générée par des suggestions négatives.

Paracétamol per os : $-0,84(95 \% \mathrm{Cl}:-0,73$ à $-0,95)[42]$

Opiacés de classe II : $-1,06(95 \% \mathrm{Cl}:-0,76$ à $-1,35)[42]$

Opiacés de classe III : $-1,20(95 \% \mathrm{Cl}:-0,6$ à $-1,8)[42]$

Suggestions négatives : $+0,74(95 \% \mathrm{Cl}:+0,4$ à $+1,08)[13]$ 